Wien klin Mag 2019 · 22:162-165

https://doi.org/10.1007/s00740-019-0293-y

Online publiziert: 20. Mai 2019

(c) Der/die Autor(en) 2019

\section{Thomas Schachner}

Universitätsklinik für Herzchirurgie, Medizinische Universität Innsbruck, Innsbruck, Österreich

\title{
Mechanische Komplikationen beim akuten Myokardinfarkt
}

\author{
Inzidenz, Diagnostik und Verlauf der \\ unterschiedlichen Formen
}

Die Inzidenz der mechanischen Komplikationen des akuten Herzinfarkts ist erfreulicherweise in den letzten Jahrzehnten nicht zuletzt durch die frühzeitige perkutane Koronarintervention zurückgegangen [1]. Sie liegt in der GröBenordnung von knapp $1 \%$ nach akutem ST-Hebungsinfarkt (STEMI). Die Sterblichkeit ist mit Größenordnungen von $50 \%$ und teilweise deutlich darüberliegend jedoch beachtlich hoch. French et al. [4] fanden in ihrer Studie bei 52 von 5745 (0,91\%) Patienten mit STEMI mechanische Komplikationen. Dies waren in $0,52 \%$ Ventrikelrupturen (Ruptur der freien Wand mit Herzbeuteltamponade), in $0,17 \%$ Ventrikelseptumrupturen (sog. Infarkt-VSD), und in 0,26\% Papillarmuskelrupturen (PMR, mit konsekutiver akuter Mitralinsuffizienz). Die 90-TageÜberlebensrate in dieser Studie war insgesamt $44 \%$, $37 \%$ für Ventrikelrupturen, $20 \%$ für Ventrikelseptumrupturen und 73,3\% für Papillarmuskelrupturen. Die mechanischen Komplikationen traten im Median 23,5h (Interquartilsabstand 5,0 bis 76,8) nach Symptombeginn auf. Diese schweren Herzinfarktkomplikationen waren für $11 \%$ der 90-TagesMortalität nach STEMI verantwortlich.

Die Gefährlichkeit der mechanischen Komplikationen nach Herzinfarkt ist darin begründet, dass sie immer zur hämodynamischen Instabilität führen, und in einem hohen Prozentsatz im kardiogenen Schock enden. In einer

Dieser Artikel basiert auf dem Vortrag $\mathrm{Ma}$ nagement mechanischer Komplikationen beim akuten Myokardinfarkt, gehalten beim 7. Intensiv- und Notfallmedizinischen Symposion am Wörthersee in Pörtschach im September 2018.
Studie an 1422 Patienten mit kardiogenem Schock nach Myokardinfarkt fanden sich in rund $12 \%$ mechanische Komplikationen des Herzinfarkts als Schockursache. Es waren in rund 7\% die akute Mitralinsuffizienz, in $4 \%$ der Infarkt-Ventrikelseptumdefekt und in etwa $1 \%$ die Tamponade [12]. Deshalb fällt auch der frühzeitigen Diagnose und Schockbekämpfung eine wesentliche Rolle in der Behandlung dieser Erkrankungsgruppe zu. Klinische Hinweise auf eine mechanische Komplikation nach Myokardinfarkt sind plötzlicher Blutdruckabfall, neue Herzgeräusche, Wiederauftreten von Thoraxschmerzen, Jugularvenenstauung und pulmonale Stauung. Eine prompte echokardiographische Untersuchung ist zur genauen Diagnose angezeigt.

Der kardiogene Schock ist definiert durch einen anhaltenden Blutdruckabfall (systolisch $<90 \mathrm{~mm} \mathrm{Hg}$ ) mit Zeichen der Hypoperfusion (Oligurie, Laktatanstieg, kalte Peripherie). Hämodynamisch finden sich eine Ruhetachykardie, ein reduzierter Herzindex $\left(<2,21 / \mathrm{min} / \mathrm{m}^{2}\right)$ und ein erhöhter Wedgedruck ( $>18 \mathrm{~mm} \mathrm{Hg})$. Echokardiografisch ist nach Herzinfarkt eine entsprechend reduzierte Linksbzw. Rechtsventrikelfunktion vorhanden [5]. Zur Behandlung eines (beginnenden) Schockzustandes bei vorhandener mechanischer Komplikation nach Myokardinfarkt ist oft eine mechanische Kreislaufunterstützung notwendig. Hierfür stehen die intraaortale Ballonpumpe (IABP), die extrakorporale Membranoxygenerierung (ECMO) und die perkutan eingebrachte Axialpumpe (Impella $\left.{ }^{\mathrm{Tx}}\right)$ zur Verfügung.

\section{Ventrikelrupturen (Ruptur der freien Wand)}

Die Ventrikelruptur ist eine seltene jedoch meist letale Komplikation des akuten Myokardinfarkts. Sie tritt in der Regel innerhalb der ersten zwei Tage nach Herzinfarkt auf. Sie tritt bei Vorderwandinfarkt in etwa gleich häufig auf wie bei Hinterwandinfarkt. Klinisch tritt ein plötzlicher Bewusstseinsverlust mit Bradykardie und Blutdruckabfall auf. Bei freier Ruptur kommt es rasch zu einer tödlichen Perikardtamponade. Im Falle einer subakuten Ventrikelruptur, im Sinne einer gedeckten Ruptur, ist bei Entwicklung eines hämodynamisch relevanten oder progredienten Perikardergusses die notfallmäßige operative Behandlung angezeigt. Diese erfolgt in der Regel durch Patchdeckung des Defekts.

\section{Papillarmuskelruptur mit konsekutiver akuter Mitralinsuffizienz}

Die Papillarmuskelruptur tritt in der Regel innerhalb einer Woche nach Herzinfarkt auf. Der hintere Papillarmuskel ist häufiger betroffen als der vordere $\mathrm{Pa}$ pillarmuskel. Es kommt konsekutiv zu einer akuten hochgradigen Mitralklappeninsuffizienz. Klinisch findet sich typischerweise eine akute ausgeprägte Dyspnoe sowie ein Lungenödem und ein neues Herzgeräusch. Die Echokardiographie führt rasch zur Diagnose. Zur hämodynamischen Stabilisierung ist aufgrund der nachlastsenkenden Wirkung die intraaortale Ballonpumpe geeignet [5]. Es 

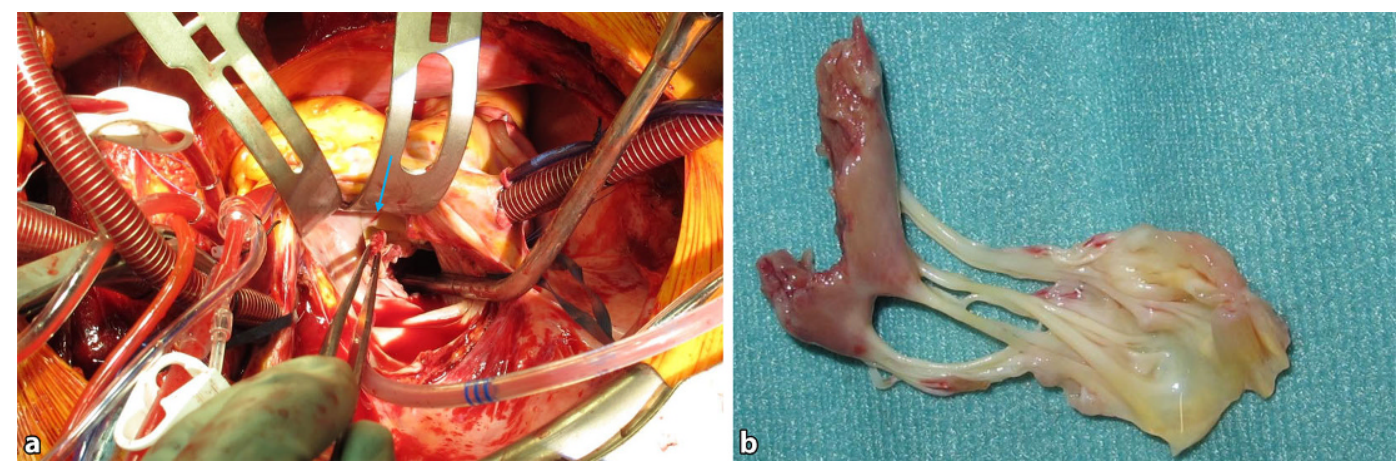

Abb. $1 \Delta$ Intraoperativer Situs bei Papillarmuskelruptur mit akuter hochgradiger Mitralinsuffizienz. a Der Patient ist an der Herz-Lungen-Maschine. Der Zugangsweg ist eine Sternotomie. Der linke Vorhof ist eröffnet und in der Pinzette wird der abgerissene Papillarmuskelteil gehalten (blauer Pfeil). Dadurch kommt es zum Prolaps großer Anteile der Mitralsegel. b Operationspräparat mit abgerissenem Papillarmuskelanteil und daran hängenden Sehnenfäden und (exzidierten) Mitralsegelteilen
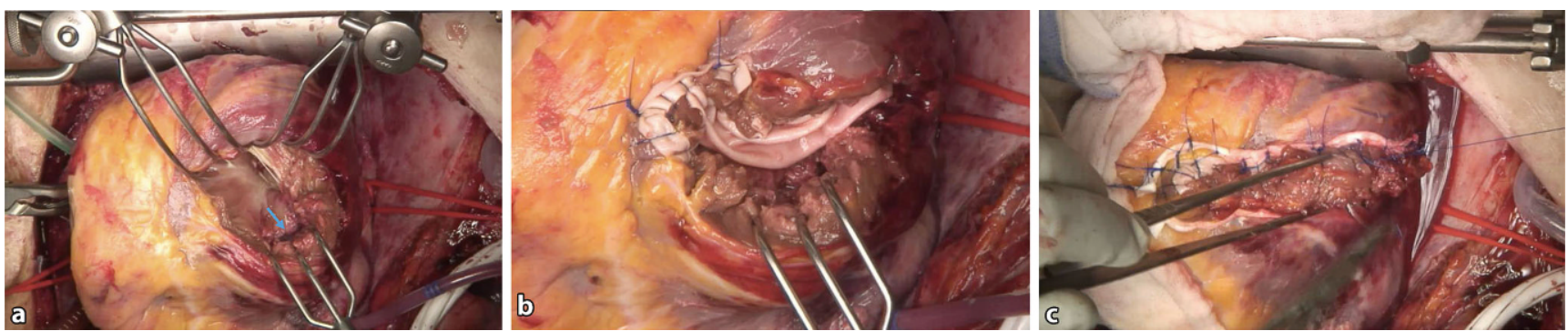

Abb. 2 \ OperativerSitus bei Infarkt-Ventrikelseptumdefekt(VSD) bei Hinterwandinfarkt. a Situs nach Ventrikulotomiedurch Infarktareal.Blauer Pfeilzeigt aufInfarkt-VSD.bZ. n. dem Einnähen eines Exklusionspatches aus bovinem Perikard.c Verschluss der Ventrikulotomie
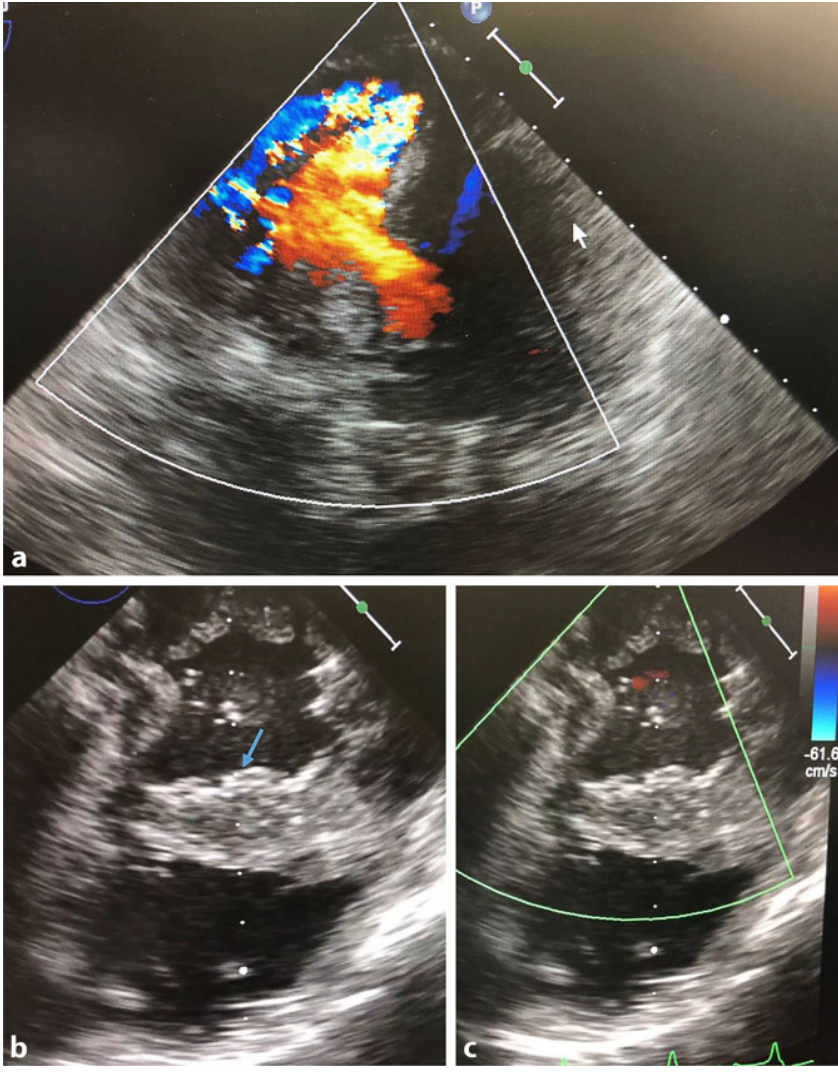

Abb. $3<$ Echokardiographie bei großem InfarktVentrikelseptumdefekt (VSD). a Infarkt-VSD mit großem Shunt im Farb-Doppler. b und $\mathbf{c}$ Zustand nach operativer Behandlung mit Exklusionspatch (blauer Pfeil, b). Im Farb-Doppler ist kein Shunt mehr detektierbar (c) gibt einzelne Fallberichte über die Verwendung einer ECMO bei Transport aus einem peripheren Spital [9] und der Impella ${ }^{\mathrm{m}}$-Pumpe [6] zur Stabilisierung bis zur operativen Behandlung. Die akute operative Behandlung erfolgt, vor allem bei kompletter Papillarmuskelruptur, in Form eines (präferenziell biologischen) Mitralklappenersatzes (vgl. • Abb. 1). Eine Studie aus dem Herzzentrum Leipzig fand bei 28 Patienten, die in den Jahren 2002-2010 aufgrund einer Papillarmuskelruptur operiert wurden, eine 30 -Tages-Mortalität von $39,3 \%$. Bei $43 \%$ der Patienten wurde präoperativ eine IABP implantiert. Eine zusätzliche koronare Bypassoperation wurde bei 66,7 \% der Patienten durchgeführt [11].

\section{Infarkt-Ventrikelseptumdefekt}

Der Infarkt-Ventrikelseptumdefekt (Infarkt-VSD) ist in $4 \%$ ursächlich für einen infarktbedingten kardiogenen Schock [12]. Er tritt in einem Zeitraum von weniger als $24 \mathrm{~h}$ post Infarkt bis mehrere Tage post Infarkt auf. Die damit assoziierte Letalität ist sehr hoch (30-88\%), 
und sie ist abhängig von der VSD-Größe und der Entwicklung eines kardiogenen Schocks. Die Verteilung zwischen Hinterwand und Vorderwand ist in etwa gleich [7]. Die Behandlungsmöglichkeiten sind einerseits die klassische operative Behandlung. Alternativ wurde in einzelnen Studien interventionelle Verschlüsse mit Amplatzer-Devices durchgeführt. Bei der operativen Behandlung gibt es prinzipiell zwei Methoden: Erstens die Implantation eines Exklusionspatches (aus bovinem Perikard, - Abb. 2 und 3), der den linken Ventrikel vom InfarktVSD abgrenzt. Zweitens kann ein VSDPatchverschluss durchgeführt werden. Arnaoutakis et al. [2] haben die Daten von 2876 Patienten mit (operativ behandeltem) Infarkt-VSD der Society of Thoracic Surgeons (USA) analysiert. Die Operationen wurden in den Jahren 1999-2010 durchgeführt. Das mittlere Alter lag bei 68 Jahren. $56 \%$ der Patienten waren Männer, $65 \%$ der Patienten erhielten präoperativ eine IABP. In dieser Studie betrug die frühe Gesamtmortalität 42,9\%. Sie war jedoch stark abhängig vom Operationszeitpunkt. So betrug die Mortalität bei Operation innerhalb von 7 Tagen 54,1\%, wohingegen sie zu einem Zeitpunkt $>7$ Tagen $18,4 \%$ betrug. Hier ist natürlich anzumerken, dass bei der Patientengruppe mit Operation >7 Tage natürlich potenziell hämodynamisch stabilere Patienten dabei sind oder Patienten vor der Operation verstorben sind. Die Autoren fanden eine erhöhte Mortalität bei präoperativer Dialyse, höherem Alter, weiblichem Geschlecht, Schockzustand, präoperativer IABP (Anm. als Ausdruck einer hämodynamischen Instabilität), Mitralinsuffizienz (mittel- oder hochgradig), RedoHerzoperation und Notfallstatus [2]. Der interventionelle Verschluss des Post-Infarkt-Ventrikelseptumdefekts stellt eine noch nicht in Guidelines etablierte Behandlungsalternative dar. Calvert et al. [3] führten in einer Multicenterstudie mit 53 Patienten in $89 \%$ der Fälle die erfolgreiche Implantation ( $4 \%$ Tod bei Intervention, $7 \%$ Notfalloperation) eines Amplatzerverschlusssystems durch. Ein kompletter Shuntverschluss konnte in $23 \%$, ein partieller Shuntverschluss in $62 \%$ der Fälle erzielt werden. Bei

Wien klin Mag 2019·22:162-165 https://doi.org/10.1007/s00740-019-0293-y

(c) Der/die Autor(en) 2019

\section{T. Schachner}

Mechanische Komplikationen beim akuten Myokardinfarkt. Inzidenz, Diagnostik und Verlauf der unterschiedlichen Formen

\section{Zusammenfassung}

Mechanische Komplikationen nach STHebungsinfarkt (STEMI) treten in etwa 1\% der Fälle auf, sind aber für mehr als 10\% der STEMI-Mortalität verantwortlich. Die 90 -Tage-Mortalität liegt bei über $50 \%$. Sie treten oft schon innerhalb von $24 \mathrm{~h}$ nach Symptombeginn auf, können aber auch einige Tage nach dem Infarkt auftreten. Die Ventrikelruptur (freie Wand) erfordert bei wirksamer Perikardergussbildung die notfallmäßige operative Behandlung. Die Papillarmuskelruptur mit akuter Mitralinsuffizienz erfordert ebenso die akute operative Therapie. Eine hämodynamische Stabilisierung ist mit intraaortaler Ballonpumpe (IABP), im Einzelfall auch mit Implantation einer venoarteriellen extrakorporalen Membranoxygenierung (ECMO) möglich. Der InfarktVentrikelseptumdefekt (VSD) erfordert eine akute oder dringliche operative Behandlung nach hämodynamischer Stabilisierung. Diese kann mittels IABP oder ECMO erfolgen. Alternativ kann ein interventioneller InfarktVSD-Verschluss in Einzelfällen erwogen werden.

\section{Schlüsselwörter}

Herzinfarkt · Ventrikelseptumruptur . Ventrikelruptur · Akute Mitralinsuffizienz . Papillarmuskelruptur

\section{Mechanical Complications in Acute Myocardial Infarction. Incidence, Diagnosis and Course of Different Forms}

\section{Abstract}

Mechanical complications after myocardial infarction occur in about $1 \%$ of ST elevation myocardial infarction (STEMI) cases, but they contribute to $10 \%$ of the STEMI mortality. Overall, the 90 -day mortality is $>50 \%$. They often appear within $24 \mathrm{~h}$ of symptom onset, but can also occur several days later. In case of free wall rupture and significant hemopericardium, emergency surgical treatment is indicated. In case of papillary muscle rupture with severe mitral regurgitation, acute surgical treatment is indicated. Hemodynamic stabilization with intra-aortic balloon pump counterpulsation (IABP) or venoarterial extracorporeal membrane oxygenation (ECMO) might be indicated in distinct cases. In case of postinfarction ventricular septal defect (VSD), acute or urgent surgical treatment is indicated after hemodynamic stabilization with ECMO or IABP. Interventional closure of the postinfarction VSD might be an alternative therapeutic option in certain cases.

\section{Keywords}

Ventricular septal defect · Ventricular septum rupture $\cdot$ Ventricular rupture $\cdot$ Acute mitral regurgitation - Papillary muscle rupture
$15 \%$ der Patienten war der Shuntverschluss erfolglos. Die Hospitalmortalität lag bei $42 \%$. Auch beim Infarkt-VSD ist die hämodynamische Stabilisierung von äußerster Wichtigkeit, um ein Abgleiten in die Schockspirale zu vermeiden. Hierfür ist die intraaortale Ballonpumpe geeignet. Bei Entwicklung eines kardiogenen Schocks ist die Implantation einer venoarteriellen extrakorporalen Membranoxygenierung (ECMO) das Mittel der Wahl. Rob et al. [10] beschreiben eine Serie von 31 Patienten mit Infarkt-VSD. Sie fanden insgesamt eine 30-TagesMortalität von $55 \%$ und eine 1-JahresMortalität von $68 \%$. Fünf Patienten wur- den als nicht operabel eingestuft, Alle 5 Patienten starben innerhalb von $30 \mathrm{Ta}$ gen. $45 \%$ der Patienten entwickelten einen kardiogenen Schock (definiert als Entwicklung von Schockzeichen trotz Katecholaminunterstützung und IABP). Die 30-Tages-Mortalität Betrug bei Patienten mit kardiogenem Schock 71,4\%, die 1-Jahres-Mortalität 85,7\%. Patienten im kardiogenen Schock mit ECMOImplantation hatten bessere Überlebensraten als Patienten im Schock ohne ECMO, die nach einem Jahr zu 100\% verstorben waren.

La Torre et al. [8] berichten in ihrer Untersuchung von 5 Patienten, bei 
denen wegen Entwicklung eines kardiogenen Schocks unter laufender IABP eine Impella $^{\text {tm }}$ Mikro-Axialpumpe transfemoral zur Stabilisierung bis zur operativen Sanierung implantiert wurde. Alle 5 Patienten konnten erfolgreich bis zur operativen Therapie initial stabilisiert werden. Zwei von 5 Patienten (40\%) verstarben innerhalb von 30 Tagen.

\section{Fazit für die Praxis}

Mechanische Komplikationen nach
ST Hebungsinfarkt (STEMI) treten in
etwa $1 \%$ der Fälle auf, sind aber für
mehr als $10 \%$ der STEMI-Mortalität
verantwortlich. Die 90 -Tage-Mortali-
tät liegt bei über $50 \%$. Sie treten oft
schon innerhalb von $24 \mathrm{~h}$ nach Sym-
ptombeginn auf, können aber auch
einige Tage post Infarkt auftreten.
Die Ventrikelruptur (freie Wand)
erfordert bei wirksamer Perikar-
dergussbildung die notfallmäßige
operative Behandlung.
Die Papillarmuskelruptur mit akuter
Mitralinsuffizienz erfordert ebenso
die akute operative Therapie. Eine
hämodynamische Stabilisierung
ist mit intraaortaler Ballonpumpe
(IABP), im Einzelfall auch mit ECMO
möglich.
Der Infarkt-Ventrikelseptumdefekt
(VSD) erfordert eine akute oder
dringliche operative Behandlung
nach hämodynamischer Stabilisie-
rung. Diese kann mittels Intraaortaler
Ballonpumpe (IABP) oder Extrakorpo-
raler Membranoxigenierung (ECM0)
erfolgen. Alternativ kann ein inter-
ventioneller Infarkt-VSD Verschluss
in Einzelfällen erwogen werden.

\section{Korrespondenzadresse}

Ao. Univ. Prof. Dr. Thomas Schachner Universitätsklinik für Herzchirurgie, Medizinische Universität Innsbruck Anichstraße 35, 6020 Innsbruck, Österreich thomas.schachner@tirol-kliniken.at

Funding. Open access funding provided by University of Innsbruck and Medical University of Innsbruck.

\section{Einhaltung ethischer Richtlinien}

Interessenkonflikt. T. Schachner gibt an, dass kein Interessenkonflikt besteht.

Für diesen Beitrag wurde von dem Autor keine Studien an Menschen oder Tieren durchgeführt. Für die aufgeführten Studien gelten die jeweils dort angegebenen ethischen Richtlinien.

Open Access Dieser Artikel wird unter der Creative Commons Namensnennung 4.0 International Lizenz (http://creativecommons.org/licenses/by/4.0/deed. de) veröffentlicht, welche die Nutzung, Vervielfältigung, Bearbeitung, Verbreitung und Wiedergabe in jeglichem Medium und Format erlaubt, sofern Sie den/die ursprünglichen Autor(en) und die Quelle ordnungsgemäßnennen, einen Link zur Creative Commons Lizenz beifügen und angeben, ob Änderungen vorgenommen wurden.

\section{Literatur}

1. Anderson JL, Morrow DA (2017) Acute myocardial infarction. NEngl J Med 376:2053-2064

2. Arnaoutakis GJ, Zhao Y, George TJ, Sciortino CM, McCarthy PM, Conte JV (2012) Surgical repair of ventricular septal defect after myocardial infarction: outcomes from the society of thoracic surgeons national database. Ann Thorac Surg 94:436-444

3. Calvert PA, Cockburn J, Wynne D, Ludman P, Rana BS, Northridge D, Hildick-Smith D et al (2014) Percutaneous closure of postinfarction ventricular septal defect: in-hospital outcomes and longterm follow-up of UK experience. Circulation 129(23):2395-2402

4. French JK, Hellkamp AS, Armstrong PW, Cohen E, Kleiman NS, O'Connor CM, Mahaffey KW et al (2010) Mechanical complications after percutaneous coronary intervention in STelevation myocardial infarction (from APEX-AMI). Am JCardiol 105:59-63

5. Ibanez B, James S, Agewall S, Antunes MJ, Bucciarelli-Ducci $\mathrm{C}$, Bueno $\mathrm{H}$, Widimsky $\mathrm{P}$ et al
(2018) 2017ESC Guidelines for the management of acute myocardial infarction in patients presenting with ST-segment elevation: The Task Force for the management of acute myocardial infarction in patients presenting with ST-segment elevation of the European Society of Cardiology (ESC). Eur Heart J39:119-177

6. Jalil B, El-Kersh K, Frizzell J et al (2017) Impella percutaneous left ventricular assist device for severe acute ischaemic mitral regurgitation as a bridge to surgery. Case Reports 2017:bcr-2017219749

7. Jones BM, Kapadia SR, Smedira NG, Robich M, Tuzcu EM, Menon V, Krishnaswamy A (2014) Ventricular septal rupture complicating acute myocardial infarction: a contemporary review. Eur Heart J 35:2060-2068

8. La Torre MW, Centofanti $P$, Attisani M, Patanè F, Rinaldi M (2011) Posterior ventricular septal defect in presence of cardiogenic shock: early implantation of the impella recover LP 5.0 as a bridge to surgery. Tex Heart Inst J 38(1):42-49

9. Obadia B, Théron A, Gariboldi V, Collart F (2014) Extracorporeal membrane oxygenation as abridge to surgery for ischemic papillary muscle rupture. JThorac Cardiovasc Surg 147:82-84

10. Rob D, Špunda R, Lindner J, Rohn V, Kunstýř J, Balík M, Bělohlávek J et al (2017) A rationale for early extracorporeal membrane oxygenation in patients with postinfarction ventricular septal rupture complicated by cardiogenic shock. Eur J HeartFail 19:97-103

11. Schroeter T, Lehmann S, Misfeld M, Borger $M$, Subramanian S, Mohr FW, Bakthiary F (2013) Clinical outcome after mitral valve surgery due to ischemic papillary muscle rupture. Ann Thorac Surg 95:820-824

12. Werdan K, Ruß M, Buerke M, Engelmann L, Ferrari M, Friedrich I, Zwißler B et al (2011) Deutschösterreichische S3-Leitlinie „Infarktbedingter kardiogener Schock - Diagnose, Monitoring und Therapie". Kardiologe 5:166-224

Hinweis des Verlags. Der Verlag bleibt in Hinblick auf geografische Zuordnungen und Gebietsbezeichnungen in veröffentlichten Karten und Institutsadressen neutral.
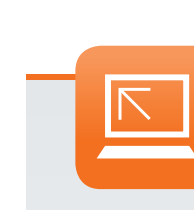

\section{Das bewegte Herz - 2019}

Integrative Bewegungsmedizin und Jubiläumstagung 30 Jahre ÖGAHM 7. - 8. November 2019 im Kurhaus, Hall in Tirol

Das Tagungsprogramm beschäftigt sich u.a mit "Herz + Lunge Interprofessionell", "Herz - Hirn - Muskel", "Gefäße + Metabolismus,", Körperliche Aktivität + strukturelle Herzerkrankungen“, „Richtig Gehen - Richtig Laufen“, „Alpin- und Höhenmedizin: Lawinenmedizin”, „Spezielle Bewegungsformen für Herzkranke“, und „Interprofessionalität in der kardiopulmonalen Rehabilitation“. Am 6. November sind Interessierte der Veranstaltung zu einem spannenden Informationsabend zum Thema: „Menschliche Extremleistungen“ eingeladen. Dabei geht es um Lebensrettung unter Extrembedingungen am Mt. Everest, Motivation Kraft - Ausdauer und Extrembergrettung in Tirol. 